\title{
Substance misuse and violence: the scope and limitations of forensic psychiatry's role
}

\author{
Peter Snowden
}

For the past 25 years, forensic psychiatry has been concerned with violent offenders with psychosis and/or personality disorder. If dual diagnosis or comorbidity meant anything to a forensic psychiatrist, it would be the 'typical' forensic case - an individual with schizophrenia and a premorbid dissocial personality who had been arrested for a violent crime. In this article I use comorbidity to describe the co-occurrence of two or more conditions (here a psychiatric disorder and health problems arising from substance misuse) rather than dual diagnosis. In fact, many violent offenders have multiple diagnoses. Williams \& Cohen (2000) argue that dual diagnosis suggests a closer relationship, perhaps including cause and effect, and is a subset of comorbidity.

Forensic psychiatrists have probably kept up with developments in criminal law as it relates to crimes associated with alcohol and drugs more than they have with the psychiatry of substance misuse (alcohol and drug misuse) and its relationship to forensic psychiatry. Speciality training arguably encourages this narrow focus. This is unfortunate, as the epidemiological catchment area (ECA) surveys (Regier et al, 1990) have shown that having a mental disorder doubles the risk of an alcohol misuse disorder and the risk increases by four times for a drugrelated disorder. The institutional ECA surveys of psychiatric patients and prisoners shows even higher levels of comorbidity, with a life-time prevalence of substance misuse disorders of $16.7 \%$ in the general population and $39 \%$ in patients of mental hospitals.

\section{The role of the modern forensic psychiatrist}

Forensic psychiatry should view the discipline of substance misuse as fundamental to practice. Practitioners should be able to make rational informed decisions when presented with offenders with a history of substance misuse, particularly when, in addition, there is a comorbid disorder (see Box 1 ).

Box 1 The role of the forensic psychiatrist

Identification To identify and assess the degree of dependence and psychological, physical and social problems associated with substance use, misuse and dependence

Decision-making To make informed decisions on how to meet the needs of the patient, based on multi-disciplinary expertise

Liaison As necessary, to contact appropriate criminal justice system services

Referral To contact other specialist services if the balance between the offender's risk to others and problems associated with the psychiatric disorder and substance misuse suggests that forensic services are not the most appropriate service to meet the individual's needs

Management To manage jointly or take a lead in managing those whose risk to others is a major concern

Peter Snowden is a consultant forensic psychiatrist and Acting Medical Director of the Mental Health Services of Salford NHS Trust (Edenfield Centre, Bury New Road, Prestwich, Manchester M25 3BL). He is a past Chair of the Forensic Faculty at the Royal College of Psychiatrists, and is a member of the Home Office Advisory Board on Restricted Patients. 


\section{Relationship of substance problems to violence and crime}

\section{Violence}

Drugs and alcohol can produce effects that may lead to violence as part of an acute psychological disturbance or as a result of misuse, withdrawal and dependence. In general, while alcohol is a major risk factor for violent offending, illicit drug use is more likely to be associated with aquisitive offending and trafficking. Athanasiadis (1999) has recently reviewed the association between drugs, alcohol and violence, in a field of research limited by methodological complexities inherent in the multifaceted nature of the problem. Not all drugs are associated with violence in populations without psychosis (see Box 2).

There is a significant relationship between early development and onset of alcohol dependence and trait aggressiveness. The aetiology of violence is complex and alcohol-induced aggression is not a uniform phenomenon. Three possible mechanisms have been proposed (Pihl \& Lemarquand, 1998) to explain the association of alcohol and violence potentiation, inhibition and disorganisation of behaviour. These might be mediated through serotonin pathways. Badawy (1998) suggests that in those with a susceptibility to aggression after alcohol consumption there is a marked depletion of brain serotonin, which may increase the likelihood of aggression in response to internal or external stimuli. Another approach has been to focus on executive cognitive functioning and it has been proposed that disruption of this system by alcohol is an underlying mechanism in alcohol-intoxicated aggression. Johns (1998) suggests that "although

Box 2 The relationship between drugs, alcohol and violence

Positive association

Cocaine and 'crack'

Amphetamines

Anabolic-androgen steroids

Alcohol

Benzodiazepines

Cannabis

No association

Sole use of opiates

Nicotine the association between substance misuse and aggression is well recognised, the mechanisms are poorly understood". Clearly, the causes are complex and are part of an interactional process involving the basic pharmacological effects of alcohol and drugs, the drinking (substance misuse) context, environment, culture and personal factors such as predisposition to aggression.

\section{Crime}

The prevalence of alcohol misuse in a survey of psychiatric morbidity of prisoners in England and Wales (Singleton et al, 1998) was 58\% for male and $36 \%$ for female remand prisoners. For the sentenced population the results were $63 \%$ and $39 \%$, respectively. Only a minority denied any past drug use. Fifty per cent of the male and $33 \%$ of the female sentenced populations were using drugs in prison. While crime and drug misuse have been high on the political agenda, there have been few initiatives to tackle the role played by alcohol in crime. The extent of the problem has been assessed by the British AllParty Group on Alcohol Misuse, which was set up in the mid-1990s to provide a forum to raise awareness in government (Touhig, 1998; Box 3).

An English special hospital study (Corbett et al, 1998) reviewed the case notes of patients admitted between 1972 and 1995. Patients with a history of substance misuse were significantly more likely to have taken illicit drugs at the time of the index offence than patients without such a history. Patients with personality disorder and a history of drug misuse were twice as likely to have taken alcohol at the time of a violent offence compared to controls.

Rasanen et al (1998) found in an unselected birth cohort study that men who misused alcohol and were diagnosed with schizophrenia were 25.2 times more likely to commit violent crime than well men. The risk for individuals with schizophrenia not dependent on alcohol was 3.6 times higher, and for individuals with other psychosis it was 7.7 times higher. This study also found that one-fifth of the male subjects with schizophrenia were dependent on alcohol by the age of 27 .

Other recent studies confirm that individuals with comorbidity are more likely than those with only psychosis to report offending or aggression. Steadman et al (1998) reported findings of the MacArthur Violence Risk Assessment Study, which compared violence by discharged patients with that by others living in the same community. There was no difference between patients and controls, but substance misuse was associated with an increased risk of violence in both groups. For patients, the effect was more obvious, with a 1-year rate of violence of 
Box 3 Evidence to the British All Party Group on Alcohol Misuse (Touhig, 1998)

Of the 10555 persons arrested and brought to Charing Cross police station in 1994, 17.6\% were arrested for drunkenness

In $1994,30 \%$ of the total probation case-load had severe alcohol problems, and the last offence of $70 \%$ of these individuals was alcohol related

In $44 \%$ of all violent incidents, the victim describes the assailant as drunk at the time

Alcohol use is associated with $60-70 \%$ of homicides, $70 \%$ of stabbings, $70 \%$ of beatings and $50 \%$ of domestic fights or assaults

One-third of homicide victims are intoxicated at the time of death

$18 \%$ for major mental disorder, increasing to $31 \%$ with comorbid substance misuse. The highest risk of violence $(43 \%)$ occurred with a combination of substance misuse and personality or adjustment disorder. A significant risk of violent behaviour has also been reported in those with dual diagnosis complicated by medication non-compliance. Thomson (1999) describes the synergistic effect between substance misuse and mental disorder in the causation of violence. Swartz et al (1998) postulate three explanations for this: substance misuse may impede medication adherence, non-compliance may lead to self-medication with illicit drugs, or non-compliance and substance misuse may both result from other factors such as personality traits and low insight.

Of the 500 homicide court reports reviewed by Appleby et al (1999) in their Report on Safer Services (70\% of the homicide cases identified), 39\% had a history of alcohol misuse and in $51 \%$ alcohol was thought to have contributed to the offence. For drug misuse the figures were $35 \%$ and $18 \%$, respectively. The homicide group was further subdivided into those with a mental illness and a larger group of other individuals (i.e. those with a personality disorder) in contact with mental health services. Those with mental illness had a lower rate of drug misuse than those with no mental illness, but the same rate of alcohol misuse. Both alcohol and drugs were more likely to be implicated in homicide in the latter group.

\section{Forensic psychiatry's role: scope and limitations}

\section{Identification}

Although the primary focus of forensic psychiatry treatment services (in-patient or community) is on the violent offender with mental disorder, a wider group of offenders is initially assessed. The forensic psychiatrist has a number of opportunities to identify offenders who misuse substances (whether or not the individual is violent) through contact with the probation service and prison health care service and through cases referred for assessment by the legal profession and the courts. Box 4 describes in general terms the purpose of any assessment by a psychiatrist of an individual who misuses drugs and alcohol. It is important to differentiate between recreational use, harmful use and a dependence syndrome. To make a definite diagnosis of dependence in ICD-10, three or more of the following should have been experienced or exhibited some time during the previous year: a compulsion to take the drug, an impaired capacity to control use, a physiological withdrawal state, tolerance, preoccupation with substance use and a persistent use despite clear evidence of harmful effects (World Health Organization, 1992).

Brooke et al (1998), in a study based on self-report data, suggest that $23 \%$ of remand prisoners would like treatment for substance misuse. At least half of these cases have no history of contact with mental

Box 4 The purpose of assessing drug and alcohol problems

To differentiate recreational use, harmful use and dependence

To distinguish between substance-related psychological symptoms and psychiatric syndromes

To identify substance-related acute presentations

To ensure adequate risk assessment

To initiate appropriate and effective therapies safely

To refer to specialist National Health Service, voluntary or statutory services 
health services and a period in custody may be the first chance to offer treatment. Prison treatment programmes have been shown to be cost-effective, with significant reductions in recidivism rates. All offenders assessed by a forensic psychiatrist should be subjected to a full psychiatric history and mental state examination, with a focus on alcohol and substance misuse. The examination should include prescribed drugs and non-compliance with them.

\section{Decision-making and liaison}

If an individual gives a history suggestive of problem use, it must decided whether this is harmful to the physical, mental and social well-being of the individual and, if so, whether there evidence of dependence and comorbidity. Any psychiatrist working in prison, but particularly the forensic psychiatrist (because of the nature of the work), has a responsibility to encourage the development of appropriate treatment opportunities for offenders who misuse substances. The changes in the provision of health care in prison (HM Prison Service \& NHS Executive, 1999), with partnership and cooperation between the National Health Service (NHS) and prison health care at all levels, should improve the provision of therapies and the quality of treatment, which has been too reliant on rapid detoxification or no detoxification with substitute medication. There is no reason why withdrawal regimes in prison should not be equivalent to those used in NHS services. Formal links should be encouraged between each prison and voluntary sector services or local specialist substance misuse services.

\section{Referral}

For violent offenders who misuse substances (without comorbidity), the clinical aspects of any inter-agency community strategy to manage risk should involve interventions to reduce the reliance on alcohol and drugs. The role of the forensic psychiatrist is limited to the referral of these individuals to the most appropriate service (NHS, independent probation or voluntary), and to encourage, with the probation service, a multi-agency risk-management approach. As a guide, where there is evidence of harmful substance use and/or dependence, then specialist alcohol or substance misuse services should be involved and if necessary (risk and the nature of the offence will dictate this), the probation service too. For non-dependent harmful use a decision will need to be made as to which is the most appropriate agency to undertake further work, and in most cases the probation service and voluntary sector should be considered.
Therapeutic work can always begin in prison at the point of assessment. Many prisons now bring in specialist substance misuse services to develop therapeutic opportunities. In other cases voluntary sector projects provide similar services. Treatment should continue when the individual is returned to the community. Advice to the court at the time of sentencing, and liaison with an offender's general practitioner (GP), the local mental health service, addiction service and probation are all routes to ensure that a treatment recommendation is put into practice. For those who do not have a GP, efforts should be made to identify one. No individual should be 'blacklisted' as a hopeless case. The health gains associated with success, or even partial success, are too great and therapeutic pessimism should be avoided.

\section{Management of violent offenders with comorbidity}

The forensic psychiatrist will be particularly involved in cases where there is a combination of a psychiatric disorder, substance misuse and violence. Such cases have become very much part of the core work of a forensic psychiatrist in recent years (Marshall,

Box 5 Factors to consider in risk assessment for dual-diagnosis violent offenders

Index offence

Nature and severity of index offence Past offending history

Substance misuse

Alcohol history

Drug history

Nature and extent of harmful use/dependence

Treatment compliance

Insight and motivation for change

Early identification of relapse of misuse

Mental illness

Nature of the illness

Treatment compliance

Insight

Early identification of relapse of illness

Relationship of violence to

Use, misuse, withdrawal or chronic use of alcohol/drugs

Mental illness and alcohol/drug misuse 
1998). The 'typical' forensic case could now be described as a violent offender with a personality disorder that predated the development of a mental illness, complicated further by harmful substance misuse. The assessment of risk (Box 5) in these cases must consider the triangular relationship between the mental illness, violence and the drug and alcohol misuse. In some cases a period of assessment in a secure unit will be necessary, in order to improve the clinical risk assessment and to help with stabilisation and detoxification from drugs and alcohol. The clinical risk assessment will then dictate the most appropriate and safest clinical risk management strategy. These are complex cases, and the risk assessment and management of the individual will have to be revisited on a regular basis and refined over time.

Offenders who pose a significant risk to others should be managed primarily by forensic services, who would be expected to manage non-dependent harmful use (Box 6). Even in these cases specialist advice can be necessary, and in some cases other services could be involved in a secondary capacity. Those with dependence on or harmful misuse of substances will need at the very least an assessment from a specialist substance misuse service (Box 7). In other cases a shared care approach may be more suitable, but the risk management plan will need to be clear about how the two clinical problems will be dealt with and whether other services are to be involved. Each case will need an individual assessment, and the outcome of the assessment in Box 5 will dictate the most appropriate balance between forensic and other services.

High-risk cases should be managed by forensic services as in-patients and then as community patients (Snowden et al, 1999). However, the limitations of such an approach must be recognised because addiction services have much to offer. It would be unusual for a forensic team not to seek the advice of specialist addiction services in such cases. Where continuity of care is not such an issue and it is decided that the risks are better managed by a shared care approach, both forensic and local/ addiction services can be involved. It is essential in such cases that responsibilities are clear, that the focus of each service is understood and that the planned response of health services to any potential increase in risk is known. Forensic mental health

Box 6 Case example 1

A 43-year-old single male with a history of alcohol dependency kills his father, with whom he is living, in the setting of an acute psychotic episode and after a number of negative life events. Further investigation reveals that 10 years previously he had a grand mal fit and at around the same time he began to present with a paranoid psychosis. After admission to a medium secure unit, and a few days before a court date, he had his second clear grand mal fit. A computerised axial tomography scan showed clear generalised brain damage.

He was prescribed carbamazepine, primarily as an anticonvulsant, but the psychotic symptoms settled also. After much debate, and with his agreement, disulfiram was prescribed. Alongside other treatment interventions, a nurse specialist from an alcohol misuse service saw him for individual sessions. As he obtained greater leave privileges he was able to attend the specialist alcohol treatment service for group work and educational sessions. Now discharged, he is primarily supervised by the forensic community team, but he is also in regular contact with a community psychiatric nurse from the local community alcohol team.

\section{Box 7 Case example 2}

A male who developed schizophrenia in his 20 s often presented to local adult mental health services after relapse. Non-compliance and associated cannabis misuse complicated his management. His use of cannabis became heavy and harmful, and relapses became more serious because he began to set fires when unwell. After causing a serious fire he was admitted to a medium secure unit. It took some considerable time to stabilise his mental state. The forensic team worked with him to reduce his cannabis habit and to show him the risks associated with cannabis use. He is now in the community, and his illness is in remission. He cooperates with antipsychotic medication. However, he still finds it difficult to be totally abstinent from cannabis. Close supervision involving hostel staff, his mother and the multi-disciplinary forensic team, with random urine testing, has managed, so far, to keep him safe in the community. 
services cannot function and meet the needs of its patients if too isolated from general adult and specialist addiction services. It is essential for such service links to be strengthened. Effective community treatment of such patients requires careful attention to compliance with treatment, and with an integrated substance misuse and mental health approach.

\section{Service models for violent offenders with comorbidity}

A number of prospective studies have shown that treatment outcomes such as symptom levels, hospitalisation rates and stability of social functioning are worse in dual diagnosis. Also, poorer adherence to treatment, more frequent violent behaviour and probably more severe clinical and social problems are found in dual diagnosis than in psychotic illness alone (Swanson et al, 1990). To make matters worse, the problems relating to the drug misuse can be as chronic as those associated with the mental illness.

Drake et al (1998) have reviewed treatment services for dual-diagnosis patients in the USA. By the 1980s many of these patients were shunted from general services to specialist substance misuse services, and were often excluded from both systems. For violent offenders with dual diagnosis this could be catastrophic, and it led to the development of integrated services with a single service and clinical programme. Interventions for substance misuse and mental health were provided by the same clinical team. Service planners in the USA (Johnson, 1998) have been developing these ideas in new 'dual diagnosis' services.

Crome (1999) reviews the situation from a UK perspective in this underresearched field. The experience of services in the USA may not be

Box 8 Strategies for managing substance misuse and dual diagnosis in the National Health Service

Developing closer links between forensic and addiction services

Facilitating at all levels (including consultant) joint discussions, cross referrals, assessments and protocols

Training and supervision in addiction techniques for all forensic teams

Specialist dual-diagnosis keyworker liaison with community forensic teams appropriate for the UK, where various models could be considered (see Box 8). Nevertheless, comprehensive out-patient programmes do appear to engage patients in services and may help them reduce substance misuse and attain remission. Service effectiveness appears to be associated with assertive outreach, case management and a longitudinal, stepwise, motivational approach to substance misuse and its treatment.

\section{Substance use and misuse in secure psychiatric units}

The use of alcohol and drugs is an increasingly important issue in the management of in-patients, even in secure units. Many patients in medium secure units have leave and can therefore bring drugs into the unit. Visitors to secure units are another source of illegal drugs and alcohol. Williams \& Cohen (2000) describe the problems on psychiatric wards. There is as yet little information about the difficulties experienced in secure units. The Fallon Inquiry into events on the personality disorder unit at Ashworth High Security Hospital describes a "system which was lax enough to allow the importation of alcohol" (Fallon et al, 1999). There is a need to draw up policies and procedures in secure units to manage these issues, but it is difficult to manage the tension between therapy and security considerations. Measures to prevent and control

Box 9 Diagnosis of substance misuse in inpatients with psychosis

The individual

Sudden unexplained exacerbation of psychosis Evidence of financial embarrassment

Intoxication

Deterioration of behaviour and violence

Victim of assault or bullying

The patients

Violence and bullying between patients

Change in mental state and behaviour of a number of patients

The ward regime

Problems following visits to the ward of specific visitors

Problems after specific patients return from leave 
drug misuse can have an oppressive effect, but drug misuse and its effects can frighten other patients and staff. This is because of violence, bullying, sexual misconduct, indebtedness and relapse (Poole \& Brabbins, 1997). Box 9 provides a helpful framework for considering the clinical issues.

\section{Conclusion}

In the past few years a series of powerful epidemiological studies (Johns, 1998) have quantified the increased risk of violent offending and the relative contributions of mental disorders and substance misuse. Substance misuse as a topic must now be viewed as central to the discipline of forensic psychiatry. The splitting of services for the offender patient with mental disorder (particularily those with psychotic illnesses) and associated substance misuse problems and the separation of training schemes for doctors and other disciplines should be reviewed if we are to provide the best services for our patients.

\section{References}

Appleby, L., Shaw, J., Amos, T., et al (1999) Safer Services: National Confidential Inquiry into Suicide and Homicide by People with Mental Illness. London: Department of Health.

Athanasiadis, L. (1999) Drugs, alcohol and violence. Current Opinion in Psychiatry, 12, 281-286.

Badawy, A. A. B. (1998) Alcohol, aggression and serotonin: metabolic aspects. Alcohol and Alcoholism, 33, 66-72.

Brooke, D., Taylor, C., Gunn, J., et al (1998) Substance misusers in prison - a treatment opportunity? Addiction, 93, 1851-1856.

Corbett, M., Duggan, C. \& Larkin, E. (1998) Substance abuse and violence: a comparison of special hospital in-patients diagnosed with either schizophrenia or personality disorder. Criminal Behaviour and Mental Health, 8, 311-321.

Crome, I. B. (1999) Substance misuse and psychiatric comorbidity: towards improved service provision. Drugs: Education, Prevention and Policy, 6, 151-174.

Drake, R. E., Mercer-McFadden, C., Meuser, K. M., et al (1998) Review of integrated mental health and substance abuse treatment for patients with dual disorders. Schizophrenia Bulletin, 24, 589-608.

Fallon, P., Bluglass, R., Edwards, B., et al (1999) Report of the Committee of Inquiry into the Personality Disorder Unit, Ashworth Special Hospital. Cm 4195. London: Stationery Office.

HM Prison Service \& NHS Executive (1999) The Future Organisation of Prison Healthcare: Report by the Joint Prison Service and National Health Service Executive Working Group. London: Department of Health.

Johns, A. (1998) Substance misuse and offending. Current Opinion in Psychiatry, 11, 669-673.

Johnson, S. (1998) The implications of dual diagnosis for service provision. The Mental Health Review, 3, 14-19.

Marshall, J. (1998) Comordity of severe mental illness and substance misuse. Journal of Forensic Psychiatry, 9, 9-12.
Pihl, R. O. \& Lemarquand, D. (1998) Serotonin and aggression in the alcohol-aggression relationship. Alcohol and Alcoholism, 33, 55-65.

Poole, R. \& Brabbins, C. (1997) Substance misuse and psychosis. British Journal of Hospital Medicine, 58, 447-450.

Rasanen, P., Tuhonen, J., Ishanni, M., et al (1998) Schizophrenia, alcohol abuse and violent behaviour: a 26 year follow-up study of an unselected birth cohort. Schizophrenia Bulletin, 24, 437-441.

Regier, D. A., Farmer, M. E., Rae, D. S., et al (1990) Comorbidity of mental disorders with alcohol and other drug abuse. Journal of the American Medical Association, 264, 2511-2518.

Singleton, N., Meltzer, H., Gatward, R., et al (1998) Psychiatric Morbidity Among Prisoners in England and Wales. London: HMSO.

Snowden, P., McKenna, J. \& Jasper, A. (1999) Management of conditionally discharged patients and others who present similar risks in the community: integrated or parallel? Journal of Forensic Psychiatry, 10, 583-596.

Steadmann, H. J., Mulvey, E. P., Monahan, J., et al (1998) Violence by people discharged from acute psychiatric facilities and by others in the same neighbourhoods. Archives of General Psychiatry, 55, 393-401.

Swanson, J., Holzer, C. \& Ganju, V. (1990) Violence and psychiatric disorder in the community: evidence from the Epidemiological Catchment Area Survey. Hospital and Community Psychiatry, 41, 761-770.

Swartz, M. S., Swanson, J. W., Hiday, V. A., et al (1998) Violence and severe mental illness: the effects of substance abuse and non-adherence to medication. American Journal of Psychiatry, 155, 226-231.

Thomson, L. D. G. (1999) Substance abuse and criminality. Current Opinion in Psychiatry, 12, 653-657.

Touhig, D. A. (1998) British All-Party Committee view on alchohol and violence. Alcohol and Alcohlism, 33, 88-91.

Williams, R. \& Cohen, J. (2000) Substance use and misuse in psychiatric wards. A model task for clinical governance? Psychiatric Bulletin, 24, 43-46.

World Health Organization (1992) The ICD-10 Classification of Mental and Behavioural Disorders. Geneva: WHO.

\section{Multiple choice questions}

1. The following substances are associated with violence in non-psychotic populations:
a alcohol
b opiates
c amphetamines
d cannabis
e nicotine.

2. Men who misuse alcohol and have a diagnosis of schizophrenia:

a present a similar risk of violence as men with schizophrenia without alcohol misuse

$b$ present with a much higher risk of violence than individuals with personality disorder and alcohol misuse

c commonly present with medication noncompliance

d should not be diagnosed as suffering from a dependence syndrome if they meet the criteria for a diagnosis of schizophrenia

e should be asked about misuse of other substances. 
3. The following criteria must be considered for an ICD-10 diagnosis of a dependence syndrome:

a compulsion to take the substance

b a psychological withdrawal state

c increased tolerance

d offending

e depression.

4. For offenders with a history of alcohol misuse and violent offending, therapeutic approaches in prison can include:

a an assessment by a specialist substance misuse service

b involvement of voluntary sector counselling services

c sulfiram

d detoxification

e risk assessment.
5. Factors to consider in risk assessment of violent offenders with a diagnosis of schizophrenia and substance misuse are:

a index offence

b post-offending history

c insight

d number of first rank symptoms

e treatment compliance.

\section{MCQ answers}

$\begin{array}{llllll}1 & 2 & 3 & 4 & 5\end{array}$

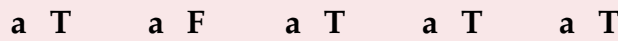

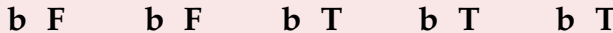

$\begin{array}{lllllllllll}c & T & \text { c } & T & \text { c } & F & \text { c } & F & \text { c } & T\end{array}$

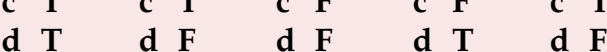

e $\begin{array}{llllllllll} & F & \text { e } & T & \text { e } & F & \text { e } & T & \text { e } & \end{array}$

\section{Commentary}

\section{John O'Grady}

Snowden (2001, this issue) comprehensively sets out the evidence for there being a strong positive link between substance misuse, mental illness and violence. He rightly points to the complexity of this interaction, which is mot confined to mentally disordered offenders and will be familiar to general psychiatrists, particularly in inner-city practice. Menezes et al (1996) provides evidence in a south London catchment area of high levels of comorbidity between serious mental illness and substance misuse, which will, as Snowden demonstrates, be linked to risk of violence. I would not disagree that, for the small number of patients with high levels of risk for violent offending (particularly those conditionally discharged under Section 41 of the Mental Health Act 1983), a parallel model of forensic care (Snowden, 1999) may be required, but even among such patients I would argue that integration with local services will be necessary to meet their complex need. For most mentally disordered offenders, there are strong arguments for an integrated model of forensic psychiatry emphasising co-working, shared care arrangements and consultation/liaison, with the emphasis placed on local service provision. What most dual-diagnosis patients, particularly dual-diagnosis mentally disordered offenders, face is formidable barriers to integrated care, and there is a risk that a 'parallel' forensic service could be one more barrier preventing patients with complex needs from gaining access to the service they require. The forensic psychiatrist's role should include close working with general psychiatry colleagues to design, commission and deliver services and service models that will meet the needs of mentally disordered offenders, particularly those with dual diagnosis. The next phase in the development of forensic psychiatry may be the creation of local services allowing proper integration between forensic, general psychiatry and substance misuse services, and prisons and other agencies involved with mentally disordered offenders (Grounds, 1996).

John C. O'Grady is a consultant forensic psychiatrist at Ravenswood House (Knowle Hospital, Fareham, Hampshire PO17 5NA). His interests include prison psychiatry, substance misuse and violent offending. 